\title{
Biologia de Ceraeochrysa claveri Navás (Neuroptera: Chrysopidae) predando Plutella xylostella (L.) (Lepidoptera: Plutellidae)
}

\author{
Biology of Ceraeochrysa claveri Navás (Neuroptera: Chrysopidae) Preying on Plutella x ylostella (L.) \\ (Lepidoptera: Plutellidae)
}

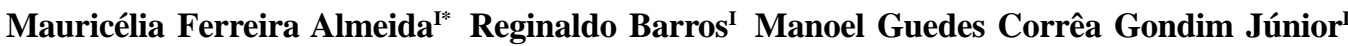 \\ Sérgio de Freitas"II Antônio Lourenço BezerraI
}

\section{RESUMO}

Ceraeochrysa claveri Navás (Neuroptera: Chrysopidae) é um predador encontrado em vários agroecossistemas, predando insetos pequenos e de tegumento mole. Seu alto potencial reprodutivo e elevada capacidade de busca o tornam capazes de predar ovos e larvas de Plutella xylostella (L.) (Lepidoptera: Plutellidae) nas partes mais internas da cabeça do repolho, onde outros inimigos naturais não conseguem. Essas características podem favorecer a utilização desse predador no controle biológico dessa praga. Neste trabalho, o objetivo é avaliar o desenvolvimento, reprodução e capacidade de predação de C. claveri, quando ovos e larvas de P. xylostella foram ofertados em condições de laboratório. Foram avaliadas as seguintes dietas: 1) ovos; 2) larvas de segundo ínstar; 3) ovos + larvas de segundo ínstar. O desenvolvimento e a viabilidade das formas imaturas de $\boldsymbol{C}$. claveri foi melhor nas dietas que continham ovos da presa. A oviposição e longevidade também foram maiores nessas dietas, independentemente da presença de larvas. Contudo, o tipo de dieta não afetou a razão sexual e o peso das larvas no oitavo dia de vida. Em média, cada larva de C. claveri foi capaz de predar 1611 ovos ou 135 larvas de segundo ínstar de $\boldsymbol{P}$. xylostella durante seu desenvolvimento. O predador foi capaz de aumentar a população 218 vezes a cada 42 dias, alimentando-se, exclusivamente, de ovos da presa. Concluiuse que o predador apresenta potencial para ser utilizado no controle biológico de P. xylostella.

Palavras-chave: tabela de vida, controle biológico, predação, traça-das-crucíferas.

\section{ABSTRACT}

Ceraeochrysa claveri Navás (Neuroptera: Chrysopidae) is a predator found in several agricultural ecosystems and it preys on small and soft body insects. Its high reproductive potential and forage capacity makes it able of preying eggs and larvae of Plutella xylostella (L.) (Lepidoptera: Plutellidae) internally hidden on cabbage head, where other natural enemies usually do not reach. These characteristics are important when choosing this predator to be used within biological control programs. This search evaluated the $\boldsymbol{C}$. claveri development, reproduction, as well as the consumption of $\boldsymbol{P}$. xylostella eggs and larvae under laboratory conditions. Therefore, it was evaluated the following diets: 1) eggs; 2) larvae of second ínstar; 3) eggs + larvae of second instar. The development and the viability of the immature forms of $\boldsymbol{C}$. claveri were better in the diets containing eggs of $\boldsymbol{P}$. xylostella. Also, the egg laying and adult longevity were greater with these diets, independently of the larvae presence. However, the diet type did not affect the sexual rate and the weight of the larvae after eight days of living. On average, each $\mathbf{C}$. claveri larva was able of preying 1611 eggs or 135 second-ínstar larvae of $\boldsymbol{P}$. xylostella during its development. The predator was able to increase its population 218 times after 42 days, feeding exclusively on P. xylostella eggs. Based on these results, this predator presents a great potential of being used within biological control programs of P. xylostella.

Key words: life table, biological control, predation, diamondback moth.

\section{INTRODUÇÃO}

Os crisopídeos, comumente conhecidos como "bichos-lixeiro", são importantes agentes reguladores de populações de artrópodes (FREITAS, 2002). Dentre os gêneros que ocorrem no Brasil, Ceraeochrysa Adams (Neuroptera: Chrysopidae)

'Universidade Federal Rural de Pernambuco (UFRPE), 52171-900, Recife, PE, Brasil. E-mail: mauricellia1@hotmail.com. *Autor para correspondência.

"Faculdade de Ciências Agrárias e Veterinárias (FCAV), Universidade Estadual Paulista (UNESP), Jaboticabal, SP, Brasil. 
contém espécies com atributos que podem torná-las predadoras importantes em vários sistemas agrícolas. Atributos como elevada capacidade de busca, alto potencial reprodutivo, capacidade de explorar habitats variados e ampla diversidade de presas (ALBUQUERQUE et al., 2001). Os crisopídeos alimentam-se de pulgões, cochonilhas, moscas-brancas, ovos e larvas de diversos outros insetos, além de vários artrópodes de pequeno tamanho e de tegumento mole (KLINGEN et al., 1996). O uso de crisopídeos como agente de controle biológico da traça-das-crucíferas Plutella xylostella (L.) (Lepidoptera: Plutellidae) pode ser uma alternativa eficiente em cultivos de repolho (TALEKAR \& SHELTON, 1993).

A traça-das-crucíferas é uma praga cosmopolita, responsável por elevados prejuízos aos produtores de repolho, tanto no Brasil (CASTELO BRANCO et al., 1996) quanto em outros países (SAYYED et al., 2002). Os danos são causados devido à alimentação da larva, que perfura as folhas, reduzindo o valor comercial do produto (BARROS et al., 1993). Uma vantagem dos crisopídeos, no controle de $\boldsymbol{P}$. xylostella, é seu elevado consumo diário de ovos e larvas (FREITAS, 2002). Comparando-se esses predadores com outros inimigos naturais de $\boldsymbol{P}$. xylostella, como Oomyzus sokolowskii (Kurdjumov) (Hymenoptera: Eulophidae), este último apresenta menor capacidade de aumento populacional, nutrindose da mesma praga (TALEKAR \& HU, 1996). Os crisopídeos também se sobressaem como predadores de $\boldsymbol{P}$. xylostella por serem capazes de predar larvas nas partes mais internas da cabeça do repolho, onde outros inimigos naturais, provavelmente, não conseguem. Finalmente, os crisopídeos têm a seu favor a existência de técnicas de criação massal em laboratório, que possibilitam a implementação de programas de controle biológico, através de liberações inundativas (VILCHEZ, 1997).

O objetivo neste trabalho foi verificar se $\boldsymbol{P}$. xylostella é uma presa adequada para o desenvolvimento e reprodução de Ceraeochrysa claveri Navás (Neuroptera: Chrysopidae) e, se o consumo daquela presa, reforça a idéia de que este crisopídeo tem potencial como inimigo natural da traçadas-crucíferas.

\section{MATERIAL E MÉTODOS}

As criações e experimentos foram realizados no Laboratório de Biologia de Insetos do Departamento de Agronomia da Universidade Federal Rural de Pernambuco(UFRPE), nas condições de $25^{\circ} \mathrm{C}$, U.R. $70 \pm 10 \%$ e fotofase de $12 \mathrm{~h}$.
Os insetos foram obtidos em plantios do repolho, Brassica oleracea (L.), var. capitata, no Município de Gravatá, PE, e criados em folhas de $\boldsymbol{B}$. oleracea var. acephala, de acordo com a metodologia descrita em BARROS \& VENDRAMIN (1999).

Adultos de C. claveri foram coletados com auxílio de rede entomológica, em casa de vegetação de produção de feijão de porco, Canavalia ensiformes (L.), no campus da UFRPE. Casais foram mantidos em gaiolas de PVC, com $20 \mathrm{~cm}$ de altura e $15 \mathrm{~cm}$ de diâmetro, revestidas, internamente, com papel sulfite branco como substrato para postura. As extremidades da gaiola foram fechadas com tecido de "naylon" presas com liga elástica, e a parte inferior apoiada em bandeja plástica. Foi fornecida, diariamente, dieta constituída de levedura de cerveja e mel, na proporção 1:1. Os ovos foram coletados diariamente e acondicionados em recipientes plásticos. Após a eclosão, as larvas foram alimentadas com ovos de Anagasta kuehniella (Zeller) (Lepidoptera: Pyralidae) obtidos de criação de laboratório, produzidos, utilizando-se dieta à base de farinha de trigo integral (60\%), farinha de milho (37\%), e levedura de cerveja (3\%), conforme TORRES et al. (1995).

Larvas da criação, com até dez horas após a eclosão, foram individualizadas em placas de Petri de 9 cm de diâmetro. Ovos e/ou larvas de P. xylostella foram fornecidos como alimento, sendo instalados três tratamentos assim constituídos: 1) ovos; 2) larvas de segundo ínstar; 3) ovos + larvas de segundo ínstar. O alimento foi fornecido, diariamente, em discos de folhas de B. oleracea var. acephala, com 230 ovos, 28 larvas ou 126 ovos + 15 larvas para cada um dos três tratamentos. As avaliações foram feitas diariamente, observando-se a duração e a viabilidade de larva, prépupa e pupa. O peso das larvas no $4^{\circ}$ e $8^{\circ}$ dias de vida foram registrados. Após a emergência, foram formados casais, individualmente, em gaiolas, alimentados conforme metodologia descrita para a criação estoque de C. claveri. Os casais foram observados diariamente, para determinação dos períodos de pré-oviposição, oviposição, pós-oviposição e número diário de ovos. Em caso de morte, os machos foram substituídos por outros da criação. Os ovos obtidos foram observados para determinação do período de incubação e viabilidade, sendo os indivíduos criados até a fase adulta para determinação da razão sexual.

Ovos ou larvas de segundo ínstar de $\boldsymbol{P}$. xylostella foram fornecidos, em discos de folha de $\boldsymbol{B}$. oleracea, var. acephala, como alimento para cada ínstar larval de $\boldsymbol{C}$. claveri, sendo instalados seis tratamentos assim constituídos: 1) 50 ovos; 2) 200 ovos; 3) 450 ovos; 4) 15 larvas; 5) 25 larvas; 6) 45 larvas. Os discos 
foram substituídos diariamente, quando se efetuava a quantificação do número de presas consumidas.

Os dados foram submetidos à análise de variância e as médias comparadas pelo teste de Tukey, utilizando o programa SANEST 3.0 (ZONTA et al., 1986). Com os dados, foram construídas tabelas de vida de fertilidade, através do pacote estatístico SAS (SAS Institute 1990), adaptando o programa escrito por MAIA et al. (2000). Esse programa utiliza o método Jackknife para estimar intervalos de confiança às médias dos tratamentos, permitindo comparações entre os pares de tratamentos pelo teste de " $\mathrm{t}$ ". Em todas as análises estatísticas, adotou-se $5 \%$ de probabilidade de erro.

\section{RESULTADOS E DISCUSSÃO}

A análise dos dados de duração e viabilidade das fases imaturas e do período de ovo a adulto de $\boldsymbol{C}$. claveri encontram-se na tabela 1. A duração do primeiro e segundo ínstar de $\boldsymbol{C}$. claveri foi prolongada com alimentação exclusiva de larvas de segundo ínstar de P. xylostella, diferindo dos tratamentos que continham ovos. Para o período de incubação e larva de terceiro ínstar, não houve diferença na duração em relação ao estágio da presa. A duração total da fase larval diferiu entre os três tratamentos, tendo variado de 9,7 dias, quando alimentado apenas com ovos, a 13,7 dias, quando o alimento foi apenas larvas, tendo a alimentação de ovos + larvas duração intermediária. A duração das fases de pré-pupa, pupa e ovo-adulto foi alongada com a alimentação exclusiva de larvas, diferindo dos tratamentos que continham ovos na dieta. A viabilidade das larvas de primeiro e segundo ínstar e do período pupal de $\boldsymbol{C}$. claveri não foi afetada pelo estágio da presa, contudo para o terceiro ínstar, período larval, pré-pupa e período de ovo-adulto, a alimentação exclusiva de larvas de $\boldsymbol{P}$. xylostella provocou a redução na viabilidade, diferindo dos tratamentos que continham ovos na dieta.

Detectou-se que os tratamentos que apresentaram ovos, em sua dieta, desenvolveram melhor o predador. Essa diferença deve-se, provavelmente, à qualidade nutricional dos ovos em comparação às larvas. Resultados obtidos por SANTACECÍLIA et al. (1997), para Ceraeochrysa cubana (Hagen), também indicaram que a qualidade do alimento ingerido pela larva também afetou o desenvolvimento.

Tabela 1 - Duração (dias) e viabilidade (\%) das fases imaturas e período de ovo a adulto de $\boldsymbol{C}$. claveri alimentado com ovos e/ou larvas de $\boldsymbol{P}$. xylostella (Temp. $25^{\circ} \mathrm{C}$, UR $70 \pm 10 \%$, fotofase $12 \mathrm{~h}$ ).

\begin{tabular}{|c|c|c|c|c|c|}
\hline C. claveri & & ovos & larvas & ovos + larvas & CV $(\%)$ \\
\hline \multirow{2}{*}{ Ovo } & duração & $3,6 \pm 0,12 \mathrm{a}^{1}$ & $3,9 \pm 0,08 \mathrm{a}$ & $3,8 \pm 0,10 \mathrm{a}$ & 11,24 \\
\hline & viabilidade & $95,0 \pm 4,99 \mathrm{a}$ & $90,0 \pm 6,88 \mathrm{a}$ & $90,0 \pm 6,88 \mathrm{a}$ & 30,82 \\
\hline \multirow{2}{*}{$1^{\circ}$ instar } & duração & $3,1 \pm 0,15 b$ & $5,4 \pm 0,28 \mathrm{a}$ & $3,4 \pm 0,11 b$ & 22,38 \\
\hline & viabilidade & $100,0 \pm 0,00 \mathrm{a}$ & $90,0 \pm 0,00 \mathrm{a}$ & $100,0 \pm 0,00 \mathrm{a}$ & 18,38 \\
\hline \multirow{2}{*}{$2^{\circ}$ ínstar } & duração & $3,4 \pm 0,11 b$ & $4,7 \pm 0,18 \mathrm{a}$ & $3,4 \pm 0,11 b$ & 15,70 \\
\hline & viabilidade & $100,0 \pm 0,00 \mathrm{a}$ & $100,0 \pm 6,88 \mathrm{a}$ & $100,0 \pm 0,00 \mathrm{a}$ & 13,54 \\
\hline \multirow{2}{*}{$3^{\circ}$ ínstar } & duração & $3,3 \pm 0,11 \mathrm{a}$ & $3,6 \pm 0,18 \mathrm{a}$ & $3,6 \pm 0,15 \mathrm{a}$ & 18,90 \\
\hline & viabilidade & $100,0 \pm 0,00 \mathrm{a}$ & $72,0 \pm 6,88 \mathrm{~b}$ & $100,0 \pm 0,00 \mathrm{a}$ & 31,98 \\
\hline \multirow{2}{*}{ Período larval } & duração & $9,7 \pm 0,16 \mathrm{c}$ & $13,7 \pm 0,23 \mathrm{a}$ & $10,4 \pm 0,18 b$ & 7,52 \\
\hline & viabilidade & $100,0 \pm 0,00 \mathrm{a}$ & $65,0 \pm 10,94 \mathrm{~b}$ & $100,0 \pm 0,00 \mathrm{a}$ & 31,98 \\
\hline \multirow{2}{*}{ Pré-pupa } & duração & $1,7 \pm 0,13 b$ & $4,2 \pm 0,39 \mathrm{a}$ & $1,7 \pm 0,13 b$ & 36,91 \\
\hline & viabilidade & $100,0 \pm 0,00 \mathrm{a}$ & $62,0 \pm 10,94 \mathrm{~b}$ & $100,0 \pm 0,00 \mathrm{a}$ & 27,39 \\
\hline \multirow{2}{*}{ Pupa } & duração & $12,0 \pm 0,09 b$ & $13,4 \pm 0,50 \mathrm{a}$ & $12,4 \pm 0,18 b$ & 6,55 \\
\hline & viabilidade & $95,0 \pm 0,00 \mathrm{a}$ & $100,0 \pm 11,24 \mathrm{a}$ & $95,0 \pm 0,00 \mathrm{a}$ & 15,27 \\
\hline \multirow{2}{*}{ Ovo-adulto } & duração & $27,0 \pm 0,30 \mathrm{~b}$ & $34,9 \pm 0,91 \mathrm{a}$ & $28,1 \pm 0,25 \mathrm{~b}$ & 22,37 \\
\hline & viabilidade & $95,0 \pm 5,00 \mathrm{a}$ & $40,0 \pm 11,24 b$ & $95,0 \pm 5,00 \mathrm{a}$ & 44,71 \\
\hline
\end{tabular}

${ }^{1}$ Médias ( \pm EP) não seguidas de mesma letra, nas linhas, diferem, significativamente, entre si pelo teste de Tukey, a 5\% de probabilidade de erro.

Ciência Rural, v.39, n.2, mar-abr, 2009. 
AUAD et al. (2001) verificaram que o estágio da presa fornecida pode interferir no desenvolvimento e sobrevivência de Ceraeochrysa cincta (Schneider), ao alimentar este predador com ovos e ninfas de Bemisia tabaci (Gennadius) biótipo B.

O peso de larvas de $\boldsymbol{C}$. claveri que receberam ovos da presa na dieta foi maior que aquelas alimentadas, exclusivamente, com larvas de $\boldsymbol{P}$. xylostella. Contudo, aos oito dias, não houve diferença (Figura 1). Segundo O'NEIL \& WIEDENMANN (1990), a massa do predador reflete seu regime alimentar. SILVA et al. (2002) afirmam que o aumento do peso pode estar relacionado à quantidade e qualidade de presas ingeridas nas 48 horas que antecederam a pesagem. $\mathrm{O}$ consumo de ovos e larvas de $\boldsymbol{P}$. xylostella por $\boldsymbol{C}$. claveri aumentou do primeiro para o terceiro ínstar (Tabela 2). No terceiro ínstar, o consumo de presas representou, aproximadamente, $80 \%$ do consumo total. Embora os crisopídeos possam predar variadas espécies de insetos em diferentes estágios de desenvolvimento, ovos de lepidópteros mostraram-se alimento adequado para a maioria das espécies estudadas (KLINGEN et al., 1996; LÓPEZ-ARROYO et al., 1999).

Fêmeas alimentadas, na fase larval com ovos na dieta apresentaram menor período de pré-oviposição e maior período de oviposição e pós-oviposição, comparadas àquelas alimentadas exclusivamente, com larvas. Contudo, não houve diferença na longevidade de fêmeas e machos, nem razão sexual em função do estágio da presa, fornecido na fase larval. $\mathrm{O}$ número de ovos/fêmea foi maior, quando o predador recebeu, na fase imatura, ovos na dieta, quando comparada a alimentação exclusiva de larvas (Tabela 3). A prévitelogênese inicia-se na fase de pupa e, para o crescimento dos ovários, a fêmea utiliza nutrientes acumulados na fase de larva, influenciando o período e a capacidade de oviposição do predador. Os maiores valores de ovos/fêmea de $\boldsymbol{C}$. claveri foram verificados em adultos provenientes de larvas alimentadas com ovos de $\boldsymbol{P}$. xylostella que, provavelmente, promoveu maior acúmulo de reservas e, consequentemente, melhor crescimento dos ovários.

A taxa líquida de reprodução foi maior, quando o predador recebeu ovos da presa na fase imatura, sendo, aproximadamente, quatro vezes maior em comparação a alimentação exclusiva de larvas de $\boldsymbol{P}$. xylostella. A taxa intrínsica de crescimento populacional e a razão finita de aumento também foram maiores, quando as dietas continham ovos da presa. De maneira inversa, o tempo médio da geração e tempo médio para a população duplicar foi maior, quando a dieta era constituída, exclusivamente, de larvas (Tabela 4). Os parâmetros da tabela de vida de fertilidade revelaram que ovos de $\boldsymbol{P}$. xylostella formam o alimento mais adequado para $\boldsymbol{C}$. claveri. Vários autores também constataram que ovos de lepidópteros constituem a presa ideal para crisopídeos (BIAGIONI \& FREITAS 2001, PESSOA et al. 2004).

\section{CONCLUSÃO}

Por meio dos valores da tabela de vida de fertilidade, pode-se concluir que $\boldsymbol{P}$. xylostella é um alimento adequado para $\boldsymbol{C}$. claveri e que esse predador apresenta potencial para ser utilizado no controle desta praga. Além disso, em condições de campo, supõe-se que $\boldsymbol{C}$. claveri se alimente dos dois estágios da presa, não comprometendo o sucesso de uma liberação, já

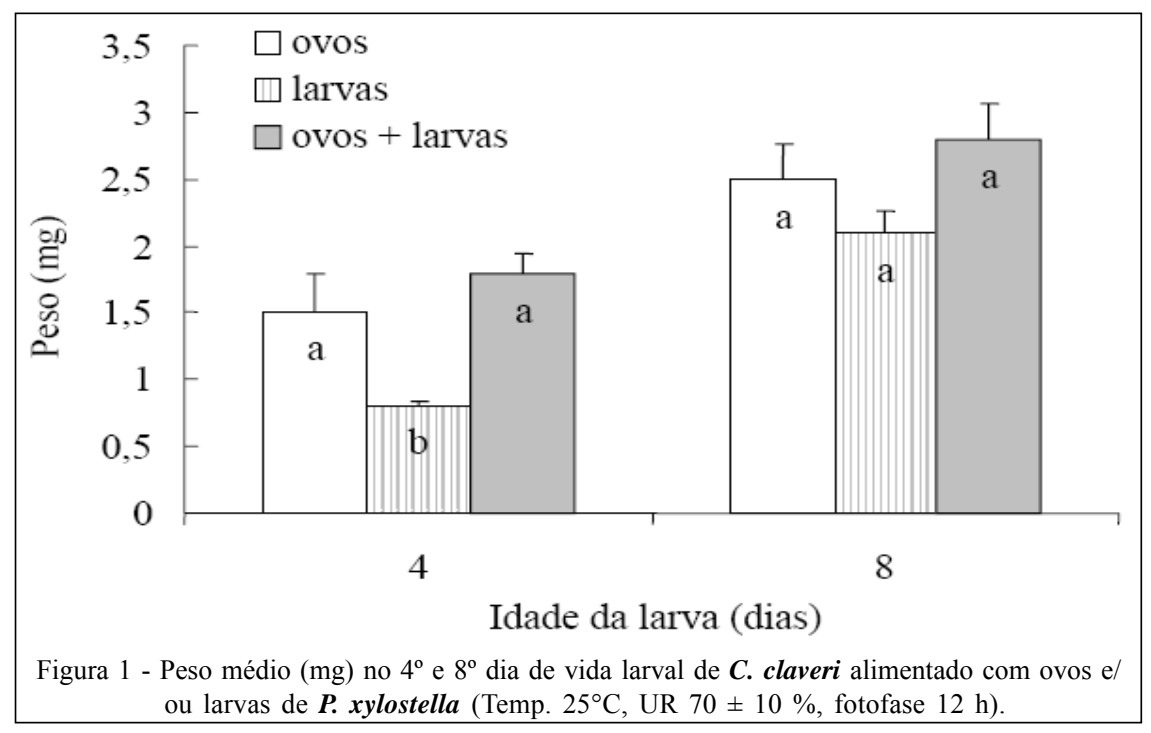

Ciência Rural, v.39, n.2, mar-abr, 2009. 
Tabela 2 - Número médio de presas consumidas por larvas de $\boldsymbol{C}$. claveri quando criadas com ovos ou larvas de $\boldsymbol{P}$. xylostella $\left(\right.$ Temp. $25^{\circ} \mathrm{C}$, UR $70 \pm 10 \%$, fotofase $12 \mathrm{~h}$ )

\begin{tabular}{|c|c|c|c|c|}
\hline \multirow{2}{*}{ C. claveri } & \multirow[b]{2}{*}{$1^{\circ}$ ínstar } & \multirow[b]{2}{*}{$2^{\circ}$ ínstar } & \multirow[b]{2}{*}{$3^{\circ}$ ínstar } & \multirow[b]{2}{*}{ Total } \\
\hline & & & & \\
\hline Ovos & $85,6 \pm 4,12 \mathrm{~d}^{1}$ & $202,4 \pm 11,73 \mathrm{c}$ & $1323,0 \pm 29,58 b$ & $1611,0 \pm 31,89 a$ \\
\hline Larvas & $13,4 \pm 1,98 \mathrm{~d}$ & $32,5 \pm 2,26 \mathrm{c}$ & $88,6 \pm 4,90 \mathrm{~b}$ & $134,5 \pm 4,94 \mathrm{a}$ \\
\hline $\mathrm{CV}(\%)$ & 28,95 & 31,72 & 13,16 & 11,47 \\
\hline
\end{tabular}

${ }^{1}$ Médias ( \pm EP) não seguidas de mesma letra, nas linhas, diferem, significativamente, entre si pelo teste de Tukey a $5 \%$ de probabilidade de erro.

Tabela 3 - Períodos reprodutivos (dias), fecundidade (número de ovos/ ㅇ) e razão sexual de $\boldsymbol{C}$. claveri, alimentado na fase larval, com ovos e/ou larvas de $\boldsymbol{P}$. xylostella (Temp. $25^{\circ} \mathrm{C}, \mathrm{UR} 70 \pm 10 \%$, fotofase $12 \mathrm{~h}$ )

\begin{tabular}{|c|c|c|c|c|}
\hline \multirow{3}{*}{ Período } & \multirow{2}{*}{\multicolumn{3}{|c|}{ P. xylostella- }} & \multirow{3}{*}{$\mathrm{CV}(\%)$} \\
\hline & & & & \\
\hline & ovos & larvas & ovos + larvas & \\
\hline Pré-oviposição & $11,0 \pm 0,45 b^{1}$ & $13,3 \pm 0,48 \mathrm{a}$ & $10,6 \pm 0,51 b$ & 9,06 \\
\hline Oviposição & $37,0 \pm 1,10 \mathrm{~b}$ & $30,3 \pm 0,75 \mathrm{a}$ & $36,2 \pm 1,46 b$ & 7,43 \\
\hline Pós-oviposição & $16,0 \pm 0,57 \mathrm{~b}$ & $24,5 \pm 1,66 \mathrm{a}$ & $18,6 \pm 0,51 b$ & 10,58 \\
\hline Longevidade 웅 & $64,0 \pm 0,84 \mathrm{a}$ & $68,0 \pm 1,46 \mathrm{a}$ & $65,4 \pm 1,96 \mathrm{a}$ & 5,13 \\
\hline Longevidade $\delta$ & $51,2 \pm 0,73 \mathrm{a}$ & $48,8 \pm 0,75 \mathrm{a}$ & $50,0 \pm 0,71 \mathrm{a}$ & 3,16 \\
\hline Fecundidade & $467,7 \pm 0,52 \mathrm{a}$ & $287,4 \pm 0,65 b$ & $456,1 \pm 0,24 \mathrm{a}$ & 12,07 \\
\hline Razão sexual & $0,49 \pm 0,00 \mathrm{a}$ & $0,48 \pm 0,01 \mathrm{a}$ & $0,49 \pm 0,00 \mathrm{a}$ & 3,42 \\
\hline
\end{tabular}

${ }^{1}$ Médias ( \pm EP) não seguidas de mesma letra, nas linhas, diferem significativamente entre si pelo teste de Tukey a $5 \%$ de probabilidade de erro.

Tabela 4 - Taxa líquida de reprodução $\left(\mathrm{R}_{\mathrm{o}}\right)$, tempo médio da geração $(\mathrm{T})$, taxa intrínsica de crescimento populacional $\left(\mathrm{r}_{\mathrm{m}}\right)$, razão finita de aumento $(\lambda)$, e tempo médio em dias para duplicar a população em números (TD) (Temp. $25^{\circ} \mathrm{C}$, UR $70 \pm 10 \%$, fotofase $12 \mathrm{~h}$ ).

\begin{tabular}{|c|c|c|c|}
\hline & ovos & larvas & ovos + larvas \\
\hline $\mathrm{R}_{\mathrm{o}}\left[\left(\right.\right.$ ( ) $\left.(\text { ( ㅇ })^{-1}\right]$ & $217,6 \pm 10,79 a^{1}$ & $55,2 \pm 4,44 \mathrm{~b}$ & $212,5 \pm 10,45 \mathrm{a}$ \\
\hline $\mathrm{T}$ (dias) & $42,6 \pm 0,46 b$ & $49,4 \pm 1,82 \mathrm{a}$ & $43,0 \pm 0,94 b$ \\
\hline$r_{m}\left[(q)(q)^{-1}(\mathrm{dia})^{-1}\right]$ & $0,126 \pm 0,002 \mathrm{a}$ & $0,081 \pm 0,002 \mathrm{~b}$ & $0,125 \pm 0,002 \mathrm{a}$ \\
\hline$\lambda\left(q / \mathrm{dia}^{-1}\right)$ & $1,13 \pm 0,002 \mathrm{a}$ & $1,08 \pm 0,003 \mathrm{~b}$ & $1,13 \pm 0,002 \mathrm{a}$ \\
\hline TD (dias) & $5,49 \pm 0,08 \mathrm{~b}$ & $8,53 \pm 0,30 \mathrm{a}$ & $5,56 \pm 0,08 b$ \\
\hline
\end{tabular}

${ }^{1}$ Médias não seguidas pela mesma letra, nas linhas, diferem entre si por meio de comparações de presas duas a duas considerando o intervalo de confiança a $5 \%$ de probabilidade de erro.

que o fornecimento de ovos isolados ou combinados com larvas ao predador, proporcionou adequado desenvolvimento, bem como não alterou a capacidade de oviposição.

\section{AGRADECIMENTOS}

À Coordenação de Aperfeiçoamento de Pessoal de Nível Superior (CAPES) e ao Conselho Nacional de
Desenvolvimento Científico e Tecnológico (CNPq), pela bolsa concedida ao primeiro autor junto ao Programa de PósGraduação em Fitossanidade/Entomologia Agrícola da UFRPE.

\section{REFERÊNCIAS}

ALBUQUeRQUe, G.S. et al. Chrysoperla externa and Ceraeochrysa spp.: potential for biological control in the New World tropics and subtropics. In: MCEWEN, P. et al.

Ciência Rural, v.39, n.2, mar-abr, 2009. 
Lacewings in the crop environment. Cambridge: Cambridge, 2001. Cap.21, p.408-423.

AUAD, A.M. et al. Aspectos biológicos dos estádios imaturos de Chrysoperla externa (Hagen) e Ceraeochrysa cincta (Schneider) (Neuroptera: Chrysopidae) alimentados com ovos e ninfas de Bemisia tabaci (Gennadius) biótipo B (Hemiptera: Aleyrodidae). Neotropical Entomology, v.30, p.429-432, 2001.

BARROS, R.; VENDRAMIM, J.D. Efeito de cultivares de repolho, utilizadas para criação de Plutella xylostella (L.) (Lepidoptera: Plutellidae), no desenvolvimento de Trichogramma pretiosum Riley (Hymenoptera: Trichogrammatidae). Anais da Sociedade Entomológica do Brasil, v.28, p.469-476, 1999.

BARROS, R. et al. Controle químico da traça-das-crucíferas Plutella xylostella (L.) (Lepidoptera: Plutellidae), em repolho. Anais da Sociedade Entomológica do Brasil, v.22, p.463469, 1993.

BIAGIONI, A.; FREITAS, S. Efeito de diferentes dietas sobre o desenvolvimento pós-embrionário de Chrysoperla defreitasi Brooks (Neuroptera: Chrysopidae). Neotropical Entomology, v.30, p.333-336, 2001.

CASTELO BRANCO, M. et al. Nível de dano de traça-dascrucíferas em repolho. Horticultura Brasileira, v.14, p.154$157,1996$.

FREITAS, S. O uso de crisopídeos no controle biológico de pragas. In: PARRA, J.R.P., et al. Controle biológico no Brasil: parasitóides e predadores. São Paulo: Manole, 2002. Cap.13, p.209-224.

KLINGEN, I. et al. The predation of Chrysoperla carnea (Neurop., Chrysopidae) on eggs and larvae of Mamestra brassicae (Lep.: Noctuidae). Journal of Applied Entomology, v.120, p.363-367, 1996.

LÓPEZ-ARROYO, J.I. et al. Effects of prey on survival, development, and reproduction of trash-carrying chrysopids (Neuroptera: Ceraeochrysa). Environmental Entomology, v.28, p.1183-1188, 1999.

MAIA, A.H.N. et al. Statistical inference on associated fertility life table parameters using Jackknife technique: computational aspects. Journal of Economic Entomology, v.93, p.511$518,2000$.
O'NEIL, R.J.; WIEDENMANN, R.N. Body weight of Podisus maculiventris under various feeding regimens. Canadian Entomology, v.122, p.285-293, 1990.

PESSOA, L.G.A. et al. Potencial reprodutivo de adultos de Chrysoperla raimundoi Freitas \& Penny (Neuroptera: Chrysopidae) em função da alimentação larval. Arquivos do Instituto Biológico, v.71, p.519-521, 2004.

SANTA-CECÍLIA, L.V.C. et al. Influência de diferentes dietas em fases imaturas de Ceraeochrysa cubana (Hagen) (Neuroptera: Chrysopidae). Anais da Sociedade Entomológica do Brasil, v.26, p.309-314, 1997.

SAS Institute. The SAS Sistem. Release 8.2. Cary NC, 1990. 890 p.

SAYYED, A.H. et al. Management of diamondback moth, Plutella xylostella (Lepidoptera: Plutellidae): a lesson from South East Asia for sustainable integrated pest management. Pakistan Journal Biological Sciences, v.5, p.234-245, 2002 .

SILVA, G.A. et al. Aspectos biológicos de Chrysoperla externa (Hagen, 1861) (Neuroptera: Chrysopidae) alimentada com lagartas de Alabama argillacea (Hübner, 1818) (Lepidoptera: Noctuidae). Ciência e Agrotecnologia, v.26, p.682-698, 2002 .

TALEKAR, N.S.; SHELTON, A.M. Biology, ecology and management of the diamondback moth. Annual Review of Entomology, v.38, p.275-301, 1993.

TALEKAR, N.S.; HU, W.J. Characteristics of parasitism of Plutella xylostella (Lep., Plutellidae) by Oomyzus sokolowiskii. Entomophaga, v.41, p.45-52, 1996.

TORRES, J.B. et al. Avaliação de diferentes porcentagens da mistura de farinha de milho com farinha de trigo integral e levedura-de-cerveja na criação de Anagasta kuehniella (Zeller, 1879). Ciência e Prática, v.19, p.365-368, 1995.

VILCHEZ, M.A.C. Metodologia de cría semiartesanal de Chrysoperla externa Hagen (Neuroptera: Chrysopidae) y su liberación en repolho para el control de Plutella xylostella L. Ceiba, Honduras, v.32, p.172, 1997.

ZONTA, E.P. et al. Sistema de análise estatística (SANEST 3.0). $P$ elotas: Instituto de Física e Matemática, UFPel, 1986. $399 \mathrm{p}$. 